Filomat 31:6 (2017), 1619-1625

DOI 10.2298/FIL1706619G

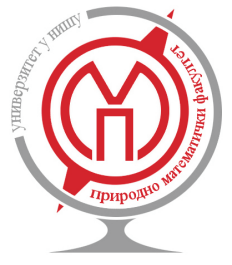

Published by Faculty of Sciences and Mathematics, University of Niš, Serbia

Available at: http://www.pmf.ni.ac.rs/filomat

\title{
Strong Convergence in Fuzzy Metric Spaces
}

\author{
Valentín Gregoria ${ }^{a}$ Juan-José Miñana ${ }^{\mathrm{b}}$ \\ ${ }^{a}$ Instituto Universitario de Matemática Pura y Aplicada, Universitat Politécnica de Valéncia, Camino de Vera s/n 46022 Valencia (SPAIN) \\ ${ }^{b}$ Departament de Ciéncies Matemátiques i Informática, Universitat de les Illes Balears, Carretera Valldemossa km. 7, 07122 Palma (SPAIN).
}

\begin{abstract}
In this paper we introduce and study the concept of strong convergence in fuzzy metric spaces $(X, M, *)$ in the sense of George and Veeramani. This concept is related with the condition $\bigwedge_{t>0} M(x, y, t)>0$, which frequently is required or missing in this context. Among other results we characterize the class of $s$-fuzzy metrics by the strong convergence defined here and we solve partially the question of finding explicitly a compatible metric with a given fuzzy metric.
\end{abstract}

\section{Introduction}

I. Kramosil and J. Michalek [10] defined the concept of fuzzy metric space which could be considered a reformulation of the concept of Menger space in fuzzy setting. This concept was modified by Grabiec in [2]. Later, George and Veeramani modified this last concept and gave a concept of fuzzy metric space $(X, M, *)$. Many concepts and results can be stated for all the above fuzzy metric spaces mentioned. In particular, if $M$ is any of these fuzzy metrics on $X$ then a topology $\tau_{M}$ deduced from $M$ is defined on $X$. A sequence $\left\{x_{n}\right\}$ in $X$ is convergent to $x_{0}$ if and only if $\lim _{n} M\left(x_{n}, x_{0}, t\right)=1$ for each $t>0$.

A significant difference between a classical metric and a fuzzy metric is that this last one includes in its definition a parameter $t$. This fact has been successfully used in engineering applications such as colour image filtering [15-17] and perceptual colour differences [5, 14]. From the mathematical point of view this parameter $t$ allows to define novel well-motivated fuzzy metric concepts which have no sense in the classical case. So, several concepts of Cauchyness and convergence have appeared in the literature (see $[2,3,6,12,18])$. Nevertheless, in some cases the natural concepts introduced are non-appropriate. A discussion of this assertion can be found in [4].

From now on by a fuzzy metric space we mean a fuzzy metric space in the sense of George and Veeramani.

Given $x, y \in X$ the real function $\left.\left.M_{x y}(t):\right] 0, \infty[\rightarrow] 0,1\right]$ defined by $M_{x y}(t)=M(x, y, t)$ is continuous in a fuzzy metric space. Notice that $M_{x y}$ is not defined at $t=0$. Then, the behaviour of $M$ for values close to 0 turns of interest. For instance, recently, for obtaining fixed point theorems for a self-mapping $T$ on $X \mathrm{D}$. Wardowski [20] and D. Mihet [13] have demanded conditions on $M$ involving $T$ for values of $t$ close to 0 . In particular, the Mihet's condition ([13, Theorem 2.4]) can be written as $\bigwedge_{t>0} M(x, T(x), t)>0$ for some $x \in X$.

2010 Mathematics Subject Classification. Primary 54A40; Secondary 54D35, 54E50

Keywords. Fuzzy metric space; s-fuzzy metric space; s-convergence

Received: 12 March 2015; Accepted: 21 May 2015

Communicated by Ljubiša D.R. Kočinac

Valentn Gregori acknowledges the support of the Spanish Ministry of Economy and Competitiveness under Grant MTM201564373-P (MINECO/FEDER, UE).

Email addresses: vgregori@mat .upv.es (Valentín Gregori), jj .minana@uib.es (Juan-José Miñana) 
This condition is related with the condition $\bigwedge_{t>0} M(x, y, t)>0$ for all $x, y \in X$, which has been studied in [6] and the obtained results are summarized in the next paragraph.

A sequence $\left\{x_{n}\right\}$ is called s-convergent to $x_{0}$ if $\lim _{n} M\left(x_{n}, x_{0}, \frac{1}{n}\right)=1$. This is a (strictly) stronger concept than convergence and it is given by a limit, which, as in the classical case, only depends on $n$. A fuzzy metric space in which every convergent sequence is $s$-convergent is called $s$-fuzzy metric space. In a similar way to the class of principal fuzzy metric spaces [3], the class of s-fuzzy metric spaces admits the following characterization by means of a special local base [6]: $(X, M, *)$ is an $s$-fuzzy metric space if and only if the family $\left\{\bigcap_{t>0} B(x, r, t): r \in\right] 0,1[\}$ is a local base at $x$, for each $x \in X$. On the other hand, if $N$ is a mapping on $X \times X$ given by $N(x, y)=\bigwedge_{t>0} M(x, y, t)$, then $(X, N, *)$ is a stationary fuzzy metric space if and only if $N(x, y)>0$ for all $x, y \in X$. In a such case, in [6] it is proved that $\tau_{N}=\tau_{M}$ if and only if $M$ is an s-fuzzy metric. However, a drawback of the concept of s-convergence, as in the case of standard Cauchy (see [4]), is that it has not a natural Cauchyness compatible pair.

The aim of this paper is to go in depth the understanding of the behaviour of a fuzzy metric $M$ when the parameter $t$ takes values close to 0 . Then, motivated by the above works, we study the behaviour of the sequential convergence when simultaneously the parameter $t$ tends to 0 . For it, we introduce a stronger concept than convergence called strong convergence, briefly st-convergence. This new concept reminds the classical concept of convergence when it is defined by the role of $\epsilon$ and $n_{0}$. So, we will say that a sequence $\left\{x_{n}\right\}$ is st-convergence to $x_{0}$ if given $\left.\epsilon \in\right] 0,1$ [ there exists $n_{0}$, depending on $\epsilon$ such that $M\left(x_{n}, x_{0}, t\right)>1-\epsilon$ for all $n \geq n_{0}$ and all $t>0$. Our first achievement is that $(X, M, *)$ is an $s$-fuzzy metric space if and only if every convergent sequence is st-convergent. Then, in Remark 3.11 we observe that for a subclass of $s$-fuzzy metrics $M$ is possible to find a compatible metric deduced explicitly from $M$. The second achievement is that the natural concept of $s t$-Cauchy sequence (Definition 4.1) deduced from st-convergence is a compatible pair, in the sense of [4] (Definition 4). This new concept fulfils also the following nice properties:

1. st-convergence implies s-convergence, and the converse is false, in general.

2. Every subsequence of a st-convergent sequence is st-convergent.

A significant difference with respect to $s$-convergence is:

3. There exist convergent sequences without st-convergent subsequences. Also:

4. In an s-fuzzy metric space Cauchy sequences are not st-Cauchy, in general.

The structure of the paper is as follows. In Section 3, after the preliminary section, we introduce and study the notion of st-convergence. In Section 4 we introduce the corresponding natural concept of stCauchyness and we show that it is compatible with st-convergence. At the end, a question related to the obtained results is proposed.

\section{Preliminaries}

Definition 2.1. (George and Veeramani [1]) A fuzzy metric space is an ordered triple $(X, M, *)$ such that $X$ is a (non-empty) set, $*$ is a continuous $t$-norm and $M$ is a fuzzy set on $X \times X \times] 0, \infty$ [ satisfying the following conditions, for all $x, y, z \in X, s, t>0$ :

(GV1) $M(x, y, t)>0$;

(GV2) $M(x, y, t)=1$ if and only if $x=y$;

(GV3) $M(x, y, t)=M(y, x, t)$;

(GV4) $M(x, y, t) * M(y, z, s) \leq M(x, z, t+s)$;

(GV5) $M(x, y,-):] 0, \infty[\rightarrow] 0,1]$ is continuous.

The continuous $t$-norms used in this paper are the usual product, denoted by $\cdot$, and the Lukasievicz $t$-norm, denoted by $\mathfrak{L}(x \mathfrak{L} y=\max \{0, x+y-1\})$, which satisfy that $\cdot \geq \mathfrak{L}$. 
Note that if $(X, M, *)$ is a fuzzy metric space and $\diamond$ is a continuous $t$-norm satisfying $\diamond \leq *$, then $(X, M, \diamond)$ is a fuzzy metric space.

If $(X, M, *)$ is a fuzzy metric space, we will say that $(M, *)$, or simply $M$, is a fuzzy metric on $X$. This terminology will be also extended along the paper in other concepts, as usual, without explicit mention.

George and Veeramani proved in [1] that every fuzzy metric $M$ on $X$ generates a topology $\tau_{M}$ on $X$ which has as a base the family of open sets of the form $\left\{B_{M}(x, \epsilon, t): x \in X, 0<\epsilon<1, t>0\right\}$, where $B_{M}(x, \epsilon, t)=\{y \in X: M(x, y, t)>1-\epsilon\}$ for all $\left.x \in X, \epsilon \in\right] 0,1[$ and $t>0$. If confusion is not possible, as usual, we write simply $B$ instead of $B_{M}$.

Let $(X, d)$ be a metric space and let $M_{d}$ a function on $\left.X \times X \times\right] 0, \infty[$ defined by

$$
M_{d}(x, y, t)=\frac{t}{t+d(x, y)}
$$

Then $\left(X, M_{d}, \cdot\right)$ is a fuzzy metric space, [1], and $M_{d}$ is called the standard fuzzy metric induced by $d$. The topology $\tau_{M_{d}}$ coincides with the topology $\tau(d)$ on $X$ deduced from $d$.

Definition 2.2. (Gregori and Romaguera [9]) A fuzzy metric $M$ on $X$ is said to be stationary if $M$ does not depend on $t$, i.e. if for each $x, y \in X$, the function $M_{x, y}(t)=M(x, y, t)$ is constant. In this case we write $M(x, y)$ instead of $M(x, y, t)$.

Proposition 2.3. (George and Veeramani [1]) Let $(X, M, *)$ a fuzzy metric space. A sequence $\left\{x_{n}\right\}$ in $X$ converges to $x$ if and only if $\lim _{n} M\left(x_{n}, x, t\right)=1$, for all $t>0$.

Definition 2.4. (George and Veeramani [1], Schweizer and Sklar [19]) A sequence $\left\{x_{n}\right\}$ in a fuzzy metric space $(X, M, *)$ is said to be $M$-Cauchy, or simply Cauchy, if for each $\epsilon \in] 0,1\left[\right.$ and each $t>0$ there is $n_{0} \in \mathbb{N}$ such that $M\left(x_{n}, x_{m}, t\right)>1-\epsilon$ for all $n, m \geq n_{0}$. Equivalently, $\left\{x_{n}\right\}$ is $M$-Cauchy if $\lim _{n, m} M\left(x_{n}, x_{m}, t\right)=1$ for all $t>0$.

As in the classical case convergent sequences are Cauchy.

Definition 2.5. (Gregori and Miñana [4]) Suppose it is given a stronger concept than convergence, say $A$-convergence. A concept of Cauchyness, say $A$-Cauchyness, is said to be compatible with $A$-convergence, and vice-versa, if the diagram of implications below is fulfilled

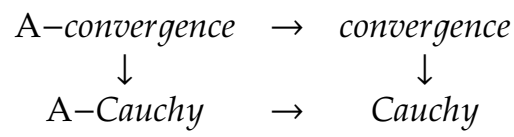

and there is not any other implication, in general, among these concepts.

From now on $(X, M, *)$, or simply $X$ if confusion is not possible, is a fuzzy metric space.

\section{Strong Convergence}

The condition of convergence in a fuzzy metric space can be rewritten as follows.

A sequence $\left\{x_{n}\right\}$ converges to $x_{0}$ if and only if for all $t>0$ and for all $\left.\epsilon \in\right] 0,1$ [ there exists $n_{\epsilon, t} \in \mathbb{N}$, depending on $\epsilon$ and $t$, such that

$$
M\left(x_{n}, x_{0}, t\right)>1-\epsilon, \text { for all } n \geq n_{\epsilon, t} .
$$

Then we can give a stronger concept than convergence strengthening in a natural way the imposition on $t$ as follows. 
Definition 3.1. A sequence $\left\{x_{n}\right\}$ in $(X, M, *)$ is strong convergent, briefly st-convergent, to $x_{0} \in X$ if given $\epsilon \in] 0,1\left[\right.$ there exists $n_{\epsilon}$, depending on $\epsilon$, such that

$$
M\left(x_{n}, x_{0}, t\right)>1-\epsilon, \text { for all } n \geq n_{\epsilon} \text { and for all } t>0 .
$$

Equivalently, $\left\{x_{n}\right\}$ is st-convergent to $x_{0} \in X$ if given $\left.\epsilon \in\right] 0,1\left[\right.$ there exists $n_{\epsilon} \in \mathbb{N}$ such that

$$
x_{n} \in B\left(x_{0}, \epsilon, t\right) \text {, for all } n \geq n_{\epsilon} \text { and for all } t>0 .
$$

Clearly, a st-convergent sequence to $x_{0}$ is convergent to $x_{0}$.

Next, we will give a characterization of st-convergent sequences by means of (double) limits.

Proposition 3.2. A sequence $\left\{x_{n}\right\}$ in $(X, M, *)$ is st-convergent to $x_{0}$ if and only if $\lim _{n, m} M\left(x_{n}, x_{0}, \frac{1}{m}\right)=1$

Proof. Suppose $\left\{x_{n}\right\}$ is st-convergent to $x_{0}$. Let $\left.\epsilon \in\right] 0,1\left[\right.$. Then we can find $n_{\epsilon}$ such that $M\left(x_{n}, x_{0}, t\right)>1-\epsilon$ for all $n \geq n_{\epsilon}$ and for all $t>0$. In particular $M\left(x_{n}, x_{0}, \frac{1}{m}\right)>1-\epsilon$ for all $n \geq n_{\epsilon}$ and for all $m \in \mathbb{N}$, i.e., $\lim _{n, m} M\left(x_{n}, x_{0}, \frac{1}{m}\right)=1$.

Conversely, suppose $\lim _{n, m} M\left(x_{n}, x_{0}, \frac{1}{m}\right)=1$. Let $\left.\epsilon \in\right] 0,1\left[\right.$. Then we can find $n_{\epsilon} \in \mathbb{N}$ such that $M\left(x_{n}, x_{0}, \frac{1}{m}\right)>1-\epsilon$ for all $n, m \geq n_{\epsilon}$. Take $t>0$. Then we can find $m_{t} \geq n_{\epsilon}$ such that $\frac{1}{m_{t}}<t$ and so $M\left(x_{n}, x_{0}, t\right) \geq M\left(x_{n}, x_{0}, \frac{1}{m_{t}}\right)>1-\epsilon$ for all $n \geq n_{\epsilon}$, so $\left\{x_{n}\right\}$ is st-convergent to $x_{0}$.

The next corollary is immediate.

Corollary 3.3. Each st-convergent sequence is s-convergent.

Now we will see that the converse of the last corollary is not true, in general.

Example 3.4. Let $\left(X, M_{d}, \cdot\right)$ be the standard fuzzy metric, where $X=\mathbb{R}$ and $d$ is the usual metric on $\mathbb{R}$.

Consider the sequence $\left\{x_{n}\right\}$, where $x_{n}=\frac{1}{n^{2}}$ for all $n \in \mathbb{N}$. The sequence $\left\{x_{n}\right\}$ is s-convergent to 0 , since

$$
\lim _{n} M_{d}\left(x_{n}, 0, \frac{1}{n}\right)=\lim _{n} \frac{\frac{1}{n}}{\frac{1}{n}+\frac{1}{n^{2}}}=1 .
$$

Now, we will see that $\left\{x_{n}\right\}$ is not st-convergent to 0 .

Suppose that $\left\{x_{n}\right\}$ is st-convergent to 0 . Then for each $\left.\epsilon \in\right] 0,1\left[\right.$ there exists $n_{\epsilon} \in \mathbb{N}$ such that $M_{d}\left(x_{n}, 0, t\right)=$ $\frac{t}{t+\frac{1}{n^{2}}}>1-\epsilon$ for all $t>0$ and for all $n \geq n_{\epsilon}$. Therefore, $\frac{1}{n_{\epsilon}^{2}}<\frac{t \epsilon}{1-\epsilon}$ for all $t>0$, a contradiction.

Under the above terminology the following assertions are immediate:

\section{Proposition 3.5.}

1. Constant sequences are st-convergent.

2. If $M$ is stationary then convergent sequences are st-convergent.

Proposition 3.6. Each subsequence of a st-convergent sequence in $\mathrm{X}$ is st-convergent.

Proof. It is straightforward.

Remark 3.7. In [6] the authors proved that in a fuzzy metric space each convergent sequence admits an $s$-convergent subsequence. This affirmation is not true for st-convergent sequences as we will show in the the next example.

Example 3.8. Consider the standard fuzzy metric space $\left(X, M_{d}, \cdot\right)$ of Example 3.4 and let $\left\{x_{n}\right\}$ be the sequence defined by $x_{n}=\frac{1}{n}$. Clearly, $\left\{x_{n}\right\}$ converges to 0 . Suppose that $\left\{x_{n_{k}}\right\}$ is a subsequence of $\left\{x_{n}\right\}$ which is stconvergent to 0 . Then for each $\epsilon \in] 0,1\left[\right.$ there exists $k_{\epsilon} \in \mathbb{N}$ such that $M_{d}\left(x_{n_{k}}, 0, t\right)=\frac{t}{t+\frac{1}{n_{k}}}>1-\epsilon$ for all $t>0$ and for all $k \geq k_{\epsilon}$. Therefore $\frac{1}{n_{k_{\epsilon}}}<\frac{t \epsilon}{1-\epsilon}$ for all $t>0$, a contradiction. 
Theorem 3.9. Every convergent sequence in $(X, M, *)$ is st-convergent if and only if every convergent sequence in $X$ is s-convergent.

Proof. If every convergent sequence in $X$ is st-convergent then by Corollary 3.3 every convergent sequence in $X$ is s-convergent.

Conversely, suppose that every convergent sequence in $X$ is s-convergent and suppose that there exists a convergent sequence $\left\{x_{n}\right\}$ to $x_{0}$ in $X$ which is not st-convergent. Then there exists $\left.\delta \in\right] 0,1[$ such that for each $k \in \mathbb{N}$ there exists $n(k) \geq k$ such that $M\left(x_{n(k)}, x_{0}, t(k)\right) \leq 1-\delta$, for some $t(k)>0$.

Next we will construct a convergent sequence $\left\{y_{j}\right\}$ which is not $s$-convergent.

Take $1 \in \mathbb{N}$, then there exists $n(1) \geq 1$ such that $M\left(x_{n(1)}, x_{0}, t(1)\right) \leq 1-\delta$. Let $n_{1} \in \mathbb{N}$ such that $n_{1} \geq \max \left\{\frac{1}{t(1)}, n(1)\right\}$ and we define

$$
y_{1}=y_{2}=\cdots=y_{n_{1}}=x_{n(1)}
$$

Now, for $n_{1} \in \mathbb{N}$, there exists $n\left(n_{1}\right) \geq n_{1}$ such that $M\left(x_{n\left(n_{1}\right)}, x_{0}, t\left(n_{1}\right)\right) \leq 1-\delta$. Let $n_{2} \in \mathbb{N}$ such that $n_{2} \geq \max \left\{\frac{1}{t\left(n_{1}\right)}, n\left(n_{1}\right)\right\}$. Clearly, $n_{2} \geq n_{1}$. So we define

$$
y_{n_{1}+1}=y_{n_{1}+2}=\cdots=y_{n_{2}}=x_{n\left(n_{1}\right)} .
$$

By induction on $k \in \mathbb{N}$, for $n_{k-1} \in \mathbb{N}$, there exists $n\left(n_{k-1}\right) \geq n_{k-1}$ such that $M\left(x_{n\left(n_{k-1}\right)}, x_{0}, t\left(n_{k-1}\right)\right) \leq 1-\delta$. Let $n_{k} \in \mathbb{N}$ such that $n_{k} \geq \max \left\{\frac{1}{t\left(n_{k-1}\right)}, n\left(n_{k-1}\right)\right\}$. Clearly, $n_{k} \geq n_{k-1}$. So we define

$$
y_{n_{k-1}+1}=y_{n_{k-1}+2}=\cdots=y_{n_{k}}=x_{n\left(n_{k-1}\right)} .
$$

The constructed sequence $\left\{y_{j}\right\}$ is convergent. Indeed, since $\left\{x_{n}\right\}$ converges to $x_{0}$ we have that for each $\epsilon \in] 0,1\left[\right.$ and $t>0$ there exists $n_{0} \in \mathbb{N}$ such that $M\left(x_{n}, x_{0}, t\right)>1-\epsilon$ for all $n \geq n_{0}$. If we take $k_{0} \in \mathbb{N}$ such that $n_{k_{0}} \geq n_{0}$ and consider $j_{0}=n_{k_{0}}$, then for each $j \geq j_{0}, y_{j}=x_{n\left(n_{k}\right)}$, where $n_{k} \geq n_{k_{0}}$, and so by construction of $\left\{y_{j}\right\}$ we have that $M\left(y_{j}, x_{0}, t\right)>1-\epsilon$.

Now, we will see that $\left\{y_{j}\right\}$ is not s-convergent to $x_{0}$. By construction of $\left\{y_{j}\right\}$ we have that for all $k \in \mathbb{N}$, $M\left(y_{n_{k}}, x_{0}, \frac{1}{n_{k}}\right) \leq 1-\delta$. Therefore there exists $\left.\delta \in\right] 0,1[$ such that for each $j \in \mathbb{N}$ we can find $k(j) \in \mathbb{N}$ such that $n_{k(j)} \geq j$ and so $M\left(y_{n_{k}(j)}, x_{0}, \frac{1}{n_{k(j)}}\right) \leq 1-\delta$. Thus $\left\{y_{j}\right\}$ is not $s$-convergent, a contradiction.

An example of $s$-fuzzy metric is (] $0, \infty[, M, \cdot)$, where $M(x, y, t)=\frac{\min \{x, y\}+t}{\max \{x, y\}+t}$. On the other hand, the standard fuzzy metric space $\left(X, M_{d}, \cdot\right)$ is s-fuzzy metric if and only if $\tau(d)$ is the discrete topology [6].

The next corollary is obvious taking into account the last theorem and Corollary 3.10 of [6].

Corollary 3.10. The following are equivalent:

(i) $M$ is an s-fuzzy metric.

(ii) $\bigcap_{t>0} B(x, r, t)$ is a neighborhood of $x$ for all $x \in X$, and for all $\left.r \in\right] 0,1[$.

(iii) $\left\{\bigcap_{t>0} B(x, r, t): r \in\right] 0,1[\}$ is a local base at $x$, for each $x \in X$.

(iv) Every convergent sequence is st-convergent.

Notice that in an s-fuzzy metric convergence can be defined with a simple limit and that one can find a local base at $x$ for each $x \in X$ depending only on the radius, which reminds the case of classical metrics. This observation is related with the next remark.

Remark 3.11. (Metric deduced explicitly from a fuzzy metric)

We will say that a metric $d$ and a fuzzy metric $M$, both on $X$, are compatible if the topologies deduced from $d$ and $M$ coincide, i.e. $\tau(d)=\tau_{M}$. Recall that a topological space is metrizable if and only if it is fuzzy metrizable [7]. Now, the topological study of a (fuzzy) metrizable space is easier thought a metric or even thought a stationary fuzzy metric because in both cases it does not appear the parameter $t$.

The reader knows that for a given metric $d$ on $X$ one can find many compatible fuzzy metrics (see [1]) deduced explicitly from $d$. The converse, up to we know, is an unsolved question. To approach this question, in the next paragraph, we recall some known results. 
Given a metric $d$ on $X$ it is easy to find stationary fuzzy metrics compatible with $d$. For instance, for a fixed $K>0$, if we define $N_{K}=\frac{K}{K+d(x, y)}$ for each $x, y \in X$ then $\left(N_{K}, \cdot\right)$ is a stationary fuzzy metric and $\tau(d)=\tau_{N_{K}}$. Conversely, if $(N, \mathfrak{I})$ is a stationary fuzzy metric on $X$ then $d(x, y)=1-N(x, y)$, for each $x, y \in X$, is a metric on $X$ and $\tau(d)=\tau_{N}$.

Now, let $* \geq \mathfrak{L}$ and suppose that $(M, *)$ is a fuzzy metric on $X$ satisfying $N(x, y)=\bigwedge_{t>0} M(x, y, t)>0$ for each $x, y \in X$. Then $(N, *)$ is a fuzzy metric on $X$ and $\tau_{N}=\tau_{M}$ if and only if $M$ is an s-fuzzy metric (see [6, Theorem 4.2]). Consequently, in this case $d(x, y)=1-\bigwedge_{t>0} M(x, y, t)$ is a metric on $X$ with $\tau(d)=\tau_{M}$ and so $d$ is a compatible metric with $M$. Clearly, $d$ is deduced explicitly from $M$.

\section{Strong Cauchy Sequences}

Next, we will give a concept of strong Cauchy sequence according to Definition 3.1.

Definition 4.1. A sequence $\left\{x_{n}\right\}$ in $X$ is strong Cauchy, briefly st-Cauchy, if given $\left.\epsilon \in\right] 0,1\left[\right.$ there exists $n_{\epsilon}$, depending on $\epsilon$, such that

$$
M\left(x_{n}, x_{m}, t\right)>1-\epsilon, \text { for all } n, m \geq n_{\epsilon} \text { and for all } t>0 .
$$

Clearly, st-Cauchy sequences are Cauchy.

In a similar way to the case of st-convergence, we give the next characterization of st-Cauchyness by means of (triple) limit.

Proposition 4.2. $\left\{x_{n}\right\}$ is st-Cauchy if and only if $\lim _{n, m, k} M\left(x_{n}, x_{m}, \frac{1}{k}\right)=1$

Proof. The proof is similar to the proof of Proposition 3.2.

We will see that the concept of st-Cauchyness is compatible with the concept of st-convergence. First, we will see that the next diagram

$$
\begin{array}{ccc}
\text { st-convergence } & \rightarrow & \text { convergence } \\
\downarrow & & \downarrow \\
s t-\text { Cauchy } & \rightarrow & \text { Cauchy }
\end{array}
$$

is fulfilled. For it, we start showing the next proposition.

Proposition 4.3. Every st-convergent sequence is st-Cauchy.

Proof. Let $\left\{x_{n}\right\}$ be a st-convergent sequence in a fuzzy metric space $(X, M, *)$. Take $\left.\epsilon \in\right] 0,1[$. By continuity of $*$, we can find $r \in] 0,1\left[\right.$ such that $(1-r) *(1-r)>1-\epsilon$. Since $\left\{x_{n}\right\}$ is st-convergent, there exists $x_{0} \in X$ and $n_{0} \in \mathbb{N}$ such that $M\left(x_{n}, x_{0}, t\right)>1-r$ for all $n \geq n_{0}$ and all $t>0$. Therefore, for each $n, m \geq n_{0}$ and each $t>0$ we have that

$$
M\left(x_{n}, x_{m}, t\right) \geq M\left(x_{n}, x_{0}, t / 2\right) * M\left(x_{0}, x_{m}, t / 2\right)>(1-r) *(1-r)>(1-\epsilon .)
$$

And thus, $\left\{x_{n}\right\}$ is st-Cauchy.

Now, we will see that the implications of the above diagram cannot be reverted in general.

Example 3.4 shows an s-convergent sequence, and so convergent, which is not st-convergent. It is easy to verify that it is also an example of convergent (Cauchy) sequence which is not st-Cauchy.

The next example shows an st-Cauchy sequence, which is not (st-)convergent.

Example 4.4. Let $(X, M, *)$ be the stationary fuzzy metric space, where $X=] 1,+\infty\left[, M(x, y)=\frac{\min \{x, y\}}{\max \{x, y\}}\right.$ and $*$ is the usual product. It is easy to verify that the sequence $\left\{x_{n}\right\}$, where $x_{n}=1+\frac{1}{n}$ is a st-Cauchy sequence in $X$, which is not (st-)convergent. 
Therefore, the concepts of st-Cauchyness and st-convergence are compatible.

Finally, we will see that in an s-fuzzy metric space Cauchy sequences are not st-Cauchy, in general.

Example 4.5. Consider $(X, M, *)$, where $X=] 0, \infty\left[, *\right.$ is the usual product and $M(x, y, t)=\frac{\min \{x, y\}+t}{\max \{x, y\}+t}$ for each $x, y \in X$ and each $t>0$. In [6] it is proved that it is an s-fuzzy metric space.

Now, if we consider the sequence $\left\{x_{n}\right\}$ in $X$, where $x_{n}=\frac{1}{n}$ for each $n \in \mathbb{N}$, it is a Cauchy sequence in $X$. Indeed,

$$
\lim _{n, m} M\left(x_{n}, x_{m}, t\right)=\lim _{n, m} \frac{\min \left\{\frac{1}{n}, \frac{1}{m}\right\}+t}{\max \left\{\frac{1}{n}, \frac{1}{m}\right\}+t}=1 .
$$

On the other hand, $\left\{x_{n}\right\}$ is not st-Cauchy. Indeed, tacking $\epsilon=\frac{1}{2}$, then for each $n \in \mathbb{N}$ we can find $m>n$ and $t>0$ such that $M\left(x_{n}, x_{m}, t\right)<\frac{1}{2}$. For instance, given $n \in \mathbb{N}$, if we consider $m=3 n$ and $\left.t \in\right] 0, \frac{1}{3 n}$ [ we have that

$$
M\left(x_{n}, x_{m}, t\right)=\frac{\frac{1}{3 n}+t}{\frac{1}{n}+t}<\frac{\frac{1}{3 n}+\frac{1}{3 n}}{\frac{1}{n}+\frac{1}{3 n}}=\frac{1}{2} .
$$

A question concerning our above study is the next.

Problem 4.6. Characterize those fuzzy metric spaces in which Cauchy sequences are st-Cauchy.

\section{References}

[1] A. George, P. Veeramani, On some results in fuzzy metric spaces, Fuzzy Sets and Systems 64 (1994) 395-399.

[2] M. Grabiec, Fixed points in fuzzy metric spaces, Fuzzy Sets and Systems 27 (1989) 385-389.

[3] V. Gregori, A. López-Crevillén, S. Morillas, A. Sapena, On convergence in fuzzy metric spaces, Topology and its Applications 156 (2009) 3002-3006.

[4] V. Gregori, J.J. Miñana, std-convergence in fuzzy metric spaces, Fuzzy Sets and Systems 267 (2015) 140-143.

[5] V. Gregori, J.J. Miñana, S. Morillas, Some questions in fuzzy metric spaces, Fuzzy Sets and Systems 204 (2012) 71-85.

[6] V. Gregori, J.J. Miñana, S. Morillas, A note on convergence in fuzzy metric spaces, Iranian Journal of Fuzzy Systems 11:4 (2014) 89-99.

[7] V. Gregori, S. Romaguera, Some properties of fuzzy metric spaces, Fuzzy Sets and Systems 115 (2000) 485-489.

[8] V. Gregori, S. Romaguera, On completion of fuzzy metric spaces, Fuzzy Sets and Systems 130 (2002) 399-404.

[9] V. Gregori, S. Romaguera, Characterizing completable fuzzy metric spaces, Fuzzy Sets and Systems 144 (2004) 411-420.

[10] I. Kramosil, J. Michalek, Fuzzy metrics and statistical metric spaces, Kybernetika 11 (1975) 326-334.

[11] K. Menger, Statistical metrics, Proceedings of the National Academy of Sciences of the United States of America 28 (1942) 535-537.

[12] D. Mihet, On fuzzy contractive mappings in fuzzy metric spaces, Fuzzy Sets and Systems 158 (2007) 915-921.

[13] D. Mihet, A note on fuzzy contractive mappings in fuzzy metric spaces, Fuzzy Sets and Systems 251 (2014) 83-91.

[14] S. Morillas, L. Gomez-Robledo, R. Huertas, M. Melgosa, Fuzzy analysis for detection of inconsistent data in experimental datasets employed at the development of the CIEDE2000 colour-difference formula, Journal of Modern Optics 56:13 (2009) 1447-1456

[15] S. Morillas, V. Gregori, A. Hervás, Fuzzy peer groups for reducing mixed gaussian impulse noise from color images, IEEE Transactions on Image Processing 18:7 (2009) 1452-1466.

[16] S. Morillas, V. Gregori, G. Peris-Fajarnés, New adaptative vector filter using fuzzy metrics, Journal of Electronic Imaging 16:3 (2007) 033007:1-15.

[17] S. Morillas, V. Gregori, G. Peris-Fajarnés, P. Latorre, A fast impulsive noise color image filter using fuzzy metrics, Real-Time Imaging 11:5-6 (2005) 417-428.

[18] L. A. Ricarte, S. Romaguera, A domain-theoretic approach to fuzzy metric spaces, Topology and its Applications 163 (2014) 149-159.

[19] B. Schweizer, A. Sklar, Probabilistic Metric Spaces, North Holland Series in Probability and Applied Mathematics, New York, Amsterdam, Oxford, 1983.

[20] D. Wardowski, Fuzzy contractive mappings and fixed points in fuzzy metric spaces, Fuzzy Sets and Systems 222 (2013) $108-114$. 